\title{
Comparable regional therapeutic effects between neoadjuvant chemotherapy and neoadjuvant chemoradiotherapy for locally advanced lower rectal cancer in terms of histopathological analysis
}

\author{
KENTARO SATO $^{1}$, TAKUYA MIURA ${ }^{1}$, SATOKO MOROHASHI ${ }^{2}$, YOSHIYUKI SAKAMOTO ${ }^{1}$, \\ HAJIME MOROHASHI ${ }^{1}$, TATSUYA YOSHIDA ${ }^{1}$ and KENICHI HAKAMADA ${ }^{1}$ \\ Departments of ${ }^{1}$ Gastroenterological Surgery, and ${ }^{2}$ Pathology and Bioscience, Hirosaki University, \\ Graduate School of Medicine, Hirosaki, Aomori 036-8562, Japan
}

Received July 30, 2018; Accepted March 18, 2019

DOI: $10.3892 / \mathrm{mco} .2019 .1835$

\begin{abstract}
Neoadjuvant chemoradiotherapy (NACRT) for lower rectal cancer is commonly used in many Western countries. NACRT improves local control, but it may also induce anal dysfunction, postoperative complications, and late effects associated with radiation. Neoadjuvant chemotherapy (NAC) for lower rectal cancer has recently been employed to improve these problems, but the local control effect of NAC when compared with NACRT is controversial. The aim of the present study was to compare the effects of NAC and NACRT using histopathological analysis. The subjects included 16 patients treated with NAC and 10 patients treated with NACRT prior to surgery. Pathological effects on primary lesions and lymph nodes were evaluated based on fibrosis and tumor depth prior to and following preoperative therapy. In the NAC and NACRT groups, the T downgrade rates were 87.5 and $80 \%$, $\mathrm{T}$ depth/F depth ratios were 0.61 and 0.73 , pathological $\mathrm{T}$ downgrade rates were 25 and $40 \%$, pathological complete response rates were 12.5 and $0 \%$ for primary lesions and 33.3 and $37.5 \%$ for lymph nodes, and the $\mathrm{N}$ conversion rates were 80 and $37.5 \%$. There were no significant differences between the groups. These results suggest that the pathological therapeutic effects of NAC were similar to those of NACRT, and NAC may be effective as an alternative therapy to NACRT.
\end{abstract}

Correspondence to: Dr Kenichi Hakamada, Department of Gastroenterological Surgery, Hirosaki University, Graduate School of Medicine, 5 Zaifu-cho, Hirosaki, Aomori 036-8562, Japan

E-mail: hakamada@hirosaki-u.ac.jp

Abbreviations: NAC, neoadjuvant chemotherapy; NACRT, neoadjuvant chemoradiotherapy; TME, total mesorectal excision; LLND, lateral lymph node dissection; pCR, pathological complete response; PRLN, pararectal lymph node; LPLN, lateral pelvic lymph node; AV, anal verge; pap, papillary adenocarcinoma; tub, tubular adenocarcinoma

Key words: rectal cancer, neoadjuvant chemotherapy, neoadjuvant chemoradiotherapy, pathological effect, fibrosis, lateral lymph node

\section{Introduction}

Many trials to reduce local recurrence in locally advanced lower rectal cancer have been reported worldwide. In Japan, the standard therapy for locally advanced rectal cancer is total mesorectal excision (TME) and lateral lymph node dissection (LLND), whereas neoadjuvant chemoradiotherapy (NACRT) combined with TME is commonly used in many Western countries. NACRT has been reported to reduce the local recurrence rate, but the prognosis is not improved (1). Furthermore, NACRT may induce anal dysfunction, postoperative complications, and late effects related to radiation (2-4). Therefore, intensive neoadjuvant chemotherapy (NAC) has recently been tried in Japan, since NAC is likely to reduce postoperative complications, maintain better anal function, and achieve early systemic control by using a strong agent, such as oxaliplatin. An advantage of NACRT is improvement of local control. Therefore, if local control of NAC is equivalent to NACRT, NAC may be useful as preoperative therapy. In this retrospective study, we compared histopathological changes and evaluated therapeutic effects in patients treated with NAC or NACRT.

\section{Patients and methods}

This retrospective study was approved by the Human Research Ethics Committee of the Hirosaki University Graduate School of Medicine (Aomori, Japan; reference no. 2017-1009). Histopathological evaluation was performed retrospectively with opt-out consent reusing previously obtained specimens for routine pathological diagnosis.

The subjects were 26 patients with locally advanced lower rectal cancer who were treated with NAC (S-1 and oxaliplatin, 3 courses; $n=16$ ) or NACRT (5-FU base chemotherapy and 40-45 Gy radiation; $n=10$ ) before surgery in our department between June 2002 and June 2016. NAC was introduced for patients participated in the clinical trial of TME and LLND after NAC [phase II study of S-1 and L-OHP neoadjuvant chemotherapy with total mesorectal excision and lateral lymph node dissection for resectable rectal cancer (ACCS-01, UMIN 000019606)] from October 2015 to June 2016. NACRT was introduced for patients with locally advanced low RC from 
June 2002 to January 2012. Patients of NACRT group were collected retrospectively. TME was performed in all cases and LLND was performed in 21 patients (15 in the NAC group and 6 in the NACRT group).

The stage was classified using the Japanese Classification of Colorectal Carcinoma 8th Edition (5). Resected specimens and lymph nodes were fixed in $10 \%$ formalin and embedded in paraffin, prior to staining with hematoxylin-eosin. In the primary tumor, we selected a slice for analysis containing the deepest residual tumor in each case. Parameters for therapeutic effects were defined based on fibrosis (Fig. 1). The invasion depth of the tumor before preoperative therapy was defined as the depth of fibrosis [distance from mucosa: F depth (mm); invasion depth: Pathological $\mathrm{F}(\mathrm{pF})]$; and the invasion depth after preoperative therapy was defined as the depth of residual tumor [distance from mucosa: $\mathrm{T}$ depth $(\mathrm{mm})$; invasion depth: Pathological T (pT)]. Measurements of these depths were performed and evaluated by two surgeons (K.S. and T.M.) and one pathologist (S.M.).

Histopathological therapeutic effects in the NAC and NACRT groups were compared using the following parameters: T downgrade rate: Improvement of invasion depth after preoperative therapy compared with clinical $\mathrm{T}$ (cT) and $\mathrm{pT}$ (example, in a case of $\mathrm{cT} 3 \rightarrow \mathrm{pT} 2, \mathrm{~T}$ downgrade was achieved); $\mathrm{T}$ depth/F depth ratio: The smaller this ratio, the greater the therapeutic effect; $\mathrm{pT}$ downgrade rate: Improvement of invasion depth after preoperative therapy compared with $\mathrm{pT}$ and $\mathrm{pF}$ (example, in a case with $\mathrm{pF}$ of $\mathrm{A}$ and $\mathrm{pT}$ of MP, pT downgrade was achieved); pathological complete response (pCR) rate: Comparison of $\mathrm{pCR}$ rate of primary legion and lymph nodes between the NAC and NACRT groups [an example of pCR for lymph nodes is shown in Fig. 1B; in lymph nodes, cases that were $\mathrm{pN}$ negative and without fibrosis were excluded (NAC: $n=6$, NACRT: $n=8)]$; and $\mathrm{N}$ negative conversion rate: Conversion rate from $\mathrm{cN}$ positive (NAC: $\mathrm{n}=10$, NACRT: $\mathrm{n}=8$ ) to pathological $\mathrm{N}(\mathrm{pN})$ negative. Statistical analyses were performed by Mann-Whitney $\mathrm{U}$ test and $\chi^{2}$ test, with $\mathrm{P}<0.05$ considered to indicate a significant difference. These analyses were performed using EZR on R commander version 1.36, programed by Kanda (6).

\section{Results}

Clinical characteristics. The clinical characteristics of the patients are shown in Table I. In the NAC group, the median distance of the tumor from the anal verge was significantly longer (5 vs. $2.5 \mathrm{~mm}, \mathrm{P}=0.01$ ) than in the NACRT group. Our department introduced laparoscopic surgery from 2014. All patients in the NACRT group were performed operation before 2014 and all patients in the NAC group were performed operation after 2014, so all operations in the NACRT group were performed by laparotomy and all operations in the NAC group were performed laparoscopically. The median operation time was significantly longer (286.5 vs. $194.5 \mathrm{~min}$, $\mathrm{P}<0.01)$ and median blood loss was significantly lower (55 vs. $546.5 \mathrm{ml}, \mathrm{P}<0.01$ ) in the NAC group. In all cases in both groups, histological types in biopsy before preoperative therapy were well or moderately differentiated adenocarcinoma. LLND was performed in 15 patients $(93.8 \%)$ in the NAC group and in $6(60 \%)$ in the NACRT group. Patients of
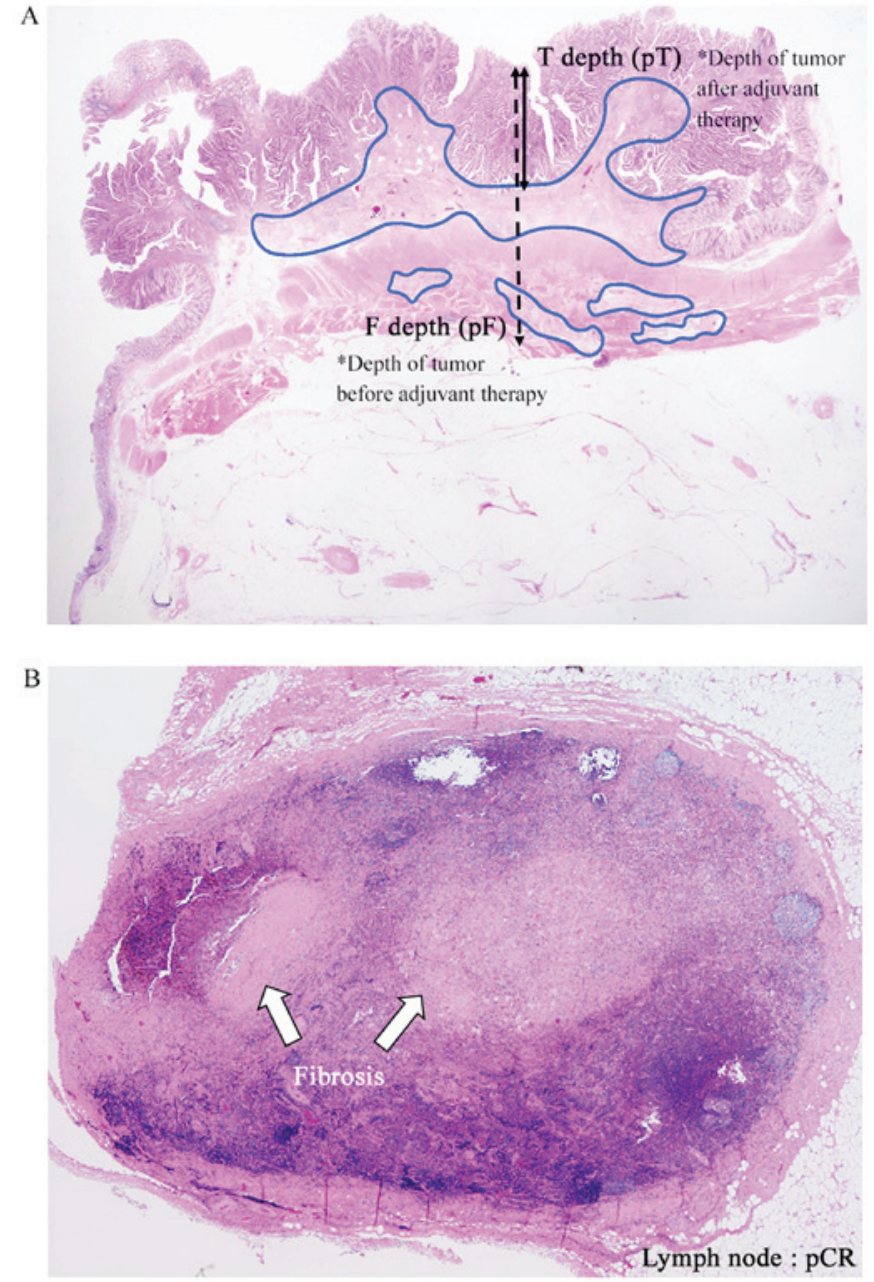

Figure 1. Hematoxylin and eosin staining. (A) Low magnification view of a hematoxylin and eosin-stained section (magnification, $x 2)$. The F depth (fibrosis) and $\mathrm{T}$ depth are the depths of the tumor from the mucosal surface prior to and following adjuvant therapy, respectively. Areas of fibrosis were surrounded with a blue outline. (B) Example of pCR for a lymph node. pCR was defined as the absence of histological evidence of vital tumor cells at the site of the primary tumor or lymph nodes and the existence of fibrosis (magnification, $\mathrm{x} 2$ ). pCR, pathological complete response.

NAC group participated in the clinical trial of LLND after NAC (ACCS-01, UMIN 000019606, described in Patients and methods), so most patients in the NAC group were performed LLND. One patient of NAC group didn't be performed lateral lymph node dissection because the lower margin of tumor didn't present in lower rectum by the results of reexaminations. In the NACRT group, LNND was performed only for patients suspected lateral pelvic lymph node (LPLN) metastasis from preoperative findings. cT grades and $\mathrm{cN}$-positive rates did not differ significantly between the groups.

Pathological characteristics. The pathological characteristics of resected specimens and lymph nodes are shown in Table II. The median $\mathrm{T}$ depth (6.0 vs. $6.8 \mathrm{~mm}, \mathrm{P}=0.75)$ and $\mathrm{F}$ depth (10.0 vs. $10.5 \mathrm{~mm}, \mathrm{P}=0.34$ ) were not significantly different between the groups. $\mathrm{pF}$ grade (invasion depth before preoperative therapy) and pT grades (invasion depth after preoperative therapy) were not significantly different between the groups ( $\mathrm{pF}, \mathrm{P}=0.05, \mathrm{pT}, \mathrm{P}=0.05)$. Positive rates of pathological metastasis to pararectal lymph nodes (PRLNs) were $18.8 \%$ in the 
Table I. Patient characteristics.

\begin{tabular}{|c|c|c|c|}
\hline Characteristic & NAC $(n=16)$ & NACRT $(n=10)$ & P-value \\
\hline Median age (range), years old & $67.5(43-77)$ & $66(53-71)$ & 0.44 \\
\hline Male, n (\%) & $14(87.5)$ & $5(50)$ & 0.07 \\
\hline Median BMI, kg/m² (range) & $21.6(17.9-29.0)$ & $22.6(17.8-25.7)$ & 0.90 \\
\hline Diabetes, n (\%) & $2(12.5)$ & $1(10)$ & 1 \\
\hline Cardiovascular disease, n (\%) & $2(12.5)$ & $1(10)$ & 1 \\
\hline Median distance of tumor from AV, $\mathrm{cm}$ (range) & $5(2-7)$ & $2.5(0-8)$ & 0.01 \\
\hline Anal preservation, n (\%) & $10(62.5)$ & $4(40)$ & 0.42 \\
\hline pap, tub, n (\%) & $16(100)$ & $10(100)$ & - \\
\hline cT4, n $(\%)$ & $8(50)$ & $5(50)$ & 1 \\
\hline cN positive, $\mathrm{n}(\%)$ & $10(62.5)$ & $8(80)$ & 0.42 \\
\hline Laparoscopic surgery, n (\%) & $16(100)$ & $0(0)$ & $<0.01$ \\
\hline Median operation time, min (range) & $286.5(249-376)$ & $194.5(149-300)$ & $<0.01$ \\
\hline Median blood loss, ml (range) & $55(3-170)$ & $546.5(105-1485)$ & $<0.01$ \\
\hline LLND, n (\%) & $15(93.8)$ & $6(60)$ & 0.05 \\
\hline
\end{tabular}

NAC, neoadjuvant chemotherapy; NACRT, neoadjuvant chemoradiotherapy; BMI, body mass index; LLND, lateral lymph node dissection; $\mathrm{AV}$, anal verge; pap, papillary adenocarcinoma; tub, tubular adenocarcinoma.

Table II. Histological features.

\begin{tabular}{lcr}
\hline Parameter & NAC $(\mathrm{n}=16)$ & NACRT $(\mathrm{n}=10)$ \\
\hline Median T depth, mm (range) & $6.0(0-19)$ & $6.8(1-23)$ \\
Median F depth, mm (range) & $10.0(3-20)$ & $10.5(5-30)$ \\
pF, n (\%) & & $0(0)$ \\
$1(\mathrm{M}-\mathrm{SM})$ & $1(6.3)$ & $1(10)$ \\
$2(\mathrm{MP})$ & $7(43.8)$ & $9(90)$ \\
$3(\mathrm{~A})$ & $7(43.8)$ & 0.75 \\
$4(\mathrm{AI})$ & $1(6.3)$ & \\
pT, n $\%)$ & & 0.34 \\
$0(\mathrm{pCR})$ & $2(12.5)$ & $3(30)$ \\
$1(\mathrm{M}-\mathrm{SM})$ & $0(0)$ & $2(20)$ \\
$2(\mathrm{MP})$ & $7(43.8)$ & $4(40)$ \\
$3(\mathrm{~A})$ & $7(43.8)$ & $1(10)$ \\
$4(\mathrm{AI})$ & $0(0)$ & 0.05 \\
pN,n $(\%)$ & & $4(40)$ \\
Pararectal $(+)$ & $3(18.8)$ & $1(16.7)$ \\
Lateral $(+)^{\mathrm{a}}$ & $0(0)$ & 0.37 \\
\hline
\end{tabular}

${ }^{a}$ LLND case alone, NAC ( $\left.n=15\right)$, NACRT (n=6). pF, pathological F; pT, pathological T; pN, pathological N; pCR, pathological complete response; M, mucosa; SM, submucosa; MP, muscularis propria; A, adventitia; AI, adjacent organ invasion.

NAC group and $40 \%$ in the NACRT group ( $\mathrm{P}=0.37)$. Positive pathological LPLN metastasis was seen in only one case in the NACRT group.

Pathological effects. The pathological effects evaluated using the five parameters described in the methods are shown in Table III. None of these parameters showed a significant difference between the NAC and NACRT groups: The T downgrade rates were good in both groups ( 87.5 vs. $80 \%, \mathrm{P}=0.63)$, and the median $\mathrm{T}$ depth/F depth ratios $(0.61$ vs. $0.73, \mathrm{P}=0.75)$ and $\mathrm{pT}$ downgrade rates $(25$ vs. $40 \%, \mathrm{P}=0.66)$ were similar. For the $\mathrm{pCR}$ rates, pCR for a primary lesion was achieved only in 2 cases in the NAC group ( 12.5 vs. $0 \%, \mathrm{P}=0.51)$, and $\mathrm{pCR}$ rates for lymph nodes were similar in the two groups ( 33 vs. $37.5 \%, \mathrm{P}=1$ ). The $\mathrm{N}$-negative conversion rate: Was slightly but not significantly higher in the NAC group ( 80 vs. $37.5 \%, \mathrm{P}=0.15$ ). 
Table III. Pathological therapeutic effects of NAC and NACRT.

\begin{tabular}{|c|c|c|c|}
\hline Parameter & NAC $(n=16)$ & NACRT $(n=10)$ & P-value \\
\hline T downgrade, $\mathrm{n}(\%)$ & $14(87.5)$ & $8(80)$ & 0.63 \\
\hline $\mathrm{T}$ depth/F depth, median (range) & $0.61(0-1.58)$ & $0.73(0.1-1)$ & 0.75 \\
\hline pT downgrade, n (\%) & $4(25)$ & $4(40)$ & 0.66 \\
\hline $\mathrm{pCR}$ rate for primary lesion, $\mathrm{n}(\%)$ & $2(12.5)$ & $0(0)$ & 0.51 \\
\hline $\mathrm{pCR}$ rate for lymph nodes, $\mathrm{n}(\%)^{\mathrm{a}}$ & $2(33.3)$ & $3(37.5)$ & 1 \\
\hline $\mathrm{N}$ negative conversion, $\mathrm{n}(\%)^{\mathrm{b}}$ & $8(80)$ & $3(37.5)$ & 0.15 \\
\hline
\end{tabular}

${ }^{a}$ NAC $(n=6)$, NACRT $(n=8)$; ${ }^{b}$ NAC $(n=10)$, NACRT $(n=8)$. NAC, neoadjuvant chemotherapy; NACRT, neoadjuvant chemoradiotherapy; pCR, pathological complete response.

Table IV. Studies of preoperative NAC and NACRT for rectal cancer.

A, NAC

\begin{tabular}{|c|c|c|c|c|c|c|}
\hline Author, year & $\mathrm{n}$ & Regimen of chemotherapy & Courses & Radiation & $\mathrm{pCR}$ rate $(\%)$ & (Refs.) \\
\hline Ishii et al, 2010 & 26 & 5-FU, LV, Irinotecan & 2 & None & 3.8 & (12) \\
\hline Hasegawa et al, 2014 & 25 & XELOX, Bevacizumab & 4 & None & 4 & (13) \\
\hline Schrag et al, 2014 & 32 & FOLFOX, Bevacizumab & 6 & None & 25 & (14) \\
\hline Kamiya et al, 2016 & 41 & XELOX & 4 & None & 12.2 & $(15)$ \\
\hline Hasegawa et al, 2017 & 60 & mFOLFOX6 & 6 & None & 16.7 & (16) \\
\hline
\end{tabular}

\section{B, NACRT}

\begin{tabular}{|c|c|c|c|c|c|c|}
\hline Author, year & $\mathrm{n}$ & Regimen of chemotherapy & Courses & Radiation & pCR rate $(\%)$ & (Refs.) \\
\hline Rödel et al, 2003 & 32 & XELOX & 2 & 50.4 Gy & 19.0 & $(17)$ \\
\hline Bosset et al, 2005 & 473 & 5-FU, LV & 5 & 45 Gy & 13.7 & $(10)$ \\
\hline Tulchinsky et al, 2008 & 132 & $5-\mathrm{FU}$ & 5 & 45-50.4 Gy & 28.0 & $(18)$ \\
\hline Roh et al, 2009 & 1,113 & 5-FU, LV & 7 & 45 Gy & 15.0 & (19) \\
\hline
\end{tabular}

Where the n number represents the number of patients who underwent surgery following NAC or NACRT, and their pCR rate was evaluated in the presented studies. NAC, neoadjuvant chemotherapy; NACRT, neoadjuvant chemoradiotherapy; pCR, pathological complete response; 5-FU, Fluorouracil; LV, leucovorin; XELOX, oxaliplatin and capecitabine; FOLFOX, folinic acid, fluorouracil and oxaliplatin.

\section{Discussion}

Many studies of multimodal therapies for locally advanced lower rectal cancer have been reported to improve outcomes. In western countries, preoperative radiotherapy and NACRT have been used for many years, and several randomized controlled trials have shown that preoperative radiotherapy and NACRT improve the local recurrence rate (7-9). Therefore, preoperative radiotherapy and NACRT combined with TME are now standard therapies for local advanced lower rectal cancer, but this approach does not improve overall survival (OS) (10). In contrast, in Japan, TME and LLND are recommended, and preoperative therapy is not included in standard therapy. However, in cases with lymph node metastasis, prognosis is poor even after LLND (11) and this has led to recent introduction of NAC in Japan. The advantages of NAC include possible improved OS by introducing intensive chemotherapy (such as FOLFOX and XELOX, including oxaliplatin) at an early stage; reduced postoperative anal dysfunction, postoperative complications, and late effects due to radiation; and a high completion rate of chemotherapy compared to postoperative adjuvant chemotherapy.

A summary of reports $(10,12-19)$ of pCR rates using NAC and NACRT is shown in Table IV. In recent reports on the effects of NAC on local control, Schrag et al (14) found a pCR rate for the primary lesion of $25 \%$ after 6 courses of FOLFOX and bevacizumab, and Kamiya et al (15) reported a pCR rate of $12.2 \%$ after 4 courses of XELOX and a rate of $31.7 \%$ for a tumor regression grade $(\mathrm{TRG}) \geq 3$. The pCR rates in these reports and our result of $12.5 \%$ for NAC are comparable with results for 5-FU-based NACRT. In Japan, SOX is sometimes used for unresectable lower RC as one of the first-line chemotherapy with Bevacizumab. On the other hands, SOX is not used as the adjuvant chemotherapy for colorectal cancer, so 
therapeutic effects of SOX for NAC and adjuvant chemotherapy is controversial. However, SOX have some good points to introduce for NAC; needless to indwell central venous catheter and port system; easy to introduce in outpatient clinic; does not induce hand foot syndrome by Capecitabine, used in XELOX regimen. Even though this study was small size, retrospective study, our result may support the effectiveness of SOX for NAC in low RC.

The pCR rate does not reveal detailed histological changes because cases exclusive of pCR are not assessed. Therefore, a more detailed pathological analysis is required in all cases. Sakuyama et al (20) evaluated histological differences between NAC and NACRT for rectal cancer, using fibrosis as a parameter for therapeutic effects. NACRT gave a smaller area of residual tumor and a shallower residual tumor depth than NAC, and ypT downstaging was more prominent with NACRT. However, NAC showed a higher $\mathrm{N}$ downgrade rate than NACRT. In our results, both NAC and NACRT had good therapeutic effects on primary lesions and lymph nodes, and the $\mathrm{N}$-negative conversion rate was higher with NAC (80\%) than with NACRT (37.5\%), although the difference was not significant.

We used the median $\mathrm{T}$ depth/F depth ratio as a parameter for therapeutic effects. This ratio became smaller when the depth of fibrosis from the mucosal surface was deeper, with a value of $<1$ indicating that the depth of fibrosis was deeper than that of the residual tumor. In our cases, the median $\mathrm{T}$ depth/F depth ratios were 0.61 with NAC and 0.73 with NACRT. Thus, the values of $<1$ suggest that both NAC and NACRT had good therapeutic effects that reached deep tissue. These results suggest that NAC and NACRT can contribute to R0 resection. The T depth/F depth ratio has not been used previously, but this parameter is useful for quantitative assessment of therapeutic effects. Sakuyama et al (20) found that NACRT had greater effects than NAC beyond the muscular layer, but in our cases the therapeutic effects of NAC and NACRT in deep regions, as evaluated by the $\mathrm{T}$ depth/F depth ratio, did not differ significantly.

This study has several limitations: The sample size was small and cases were analyzed retrospectively; the chemotherapy patients in the NAC group received (SOX) was not standard regimen in the western countries; the patient characteristics in the NAC and NACRT groups were markedly different, so selection bias may be present; and the study only assessed histological effects, and did not assess clinical outcomes, such as local recurrence rate and OS. Therefore, a randomized controlled trial of clinical outcomes of NAC and NACRT is required to compare actual clinical therapeutic effects. Within these limitations, we conclude that both NAC and NACRT are good preoperative therapy from a pathological perspective. NAC gave local control equivalent to that of NACRT, and therapeutic effects for lymph nodes might be better with NAC. These findings suggest that NAC can serve as an alternative therapy to NACRT.

\section{Acknowledgements}

Not applicable.

\section{Funding}

No funding was received.

\section{Availability of data and materials}

All data generated and analyzed in the present study are included in this published article.

\section{Authors' contributions}

KS, TM, SM, YS, HM, TY and KH all contributed to the study concept and design. KS, TM and SM carried out the pathological analysis. YS, HM and TY performed the operations. KH supervised the study. KS, TM, SM, YS, HM, TY and $\mathrm{KH}$ all participated in the interpretation of the results and the writing of the report, and all approved the final version. All authors read and approved the final manuscript.

\section{Ethics approval and consent to participate}

The present retrospective study was approved by the Human ResearchEthics Committee of the Hirosaki University Graduate School of Medicine (Aomori, Japan; reference no. 2017-1009). Histopathological evaluation was performed retrospectively with opt-out consent reusing previously obtained specimens for routine pathological diagnosis.

\section{Patient consent for publication}

Not applicable.

\section{Competing interests}

The authors declare that they have no competing interests.

\section{References}

1. De Caluwé L, Van Nieuwenhove Y and Ceelen WP: Preoperative chemoradiation versus radiation alone for stage II and III resectable rectal cancer. Cochrane Database Syst Rev 28: 2, 2013.

2. Marijnen CA, van de Velde CJ, Putter H, van den Brink M, Maas CP, Martijn H, Rutten HJ, Wiggers T, Kranenbark EK, Leer JW and Stiggelbout AM: Impact of short-term preoperative radio- therapy on health-related quality of life and sexual functioning in primary rectal cancer: Report of a multicenter randomized trial. J Clin Oncol 23: 1847-1858, 2005.

3. Camma C, Giunta M, Fiorica F, Pagliaro L, Craxì A and Cottone M: Preoperative radiotherapy for resectable rectal cancer: A meta-analysis. J Am Med Assoc 284: 1008-1015, 2000.

4. Holm T, Rutqvist LE, Johansson H and Cedermark B: Postoperative mortality in rectal cancer treated with or without preoperative radiotherapy: Causes and risk factors. Br J Surg 83: 964-968, 1996.

5. Japanese Society for Cancer of the Colon and Rectum: Japanese Classification of Colorectal Carcinoma. 8th edition. Kanehara Shuppan, Tokyo, 2013.

6. Kanda Y: Investigation of the freely available easy-to-use software 'EZR' for medical statistics. Bone Marrow Transplantat 48 , 452-458, 2013

7. Frykholm GJ, Glimelius B and Pahlman L: Preoperative or postoperative irradiation in adenocarcinoma of the rectum: Final treatment results of a randomized trial and an evaluation of late secondary effects. Dis Colon Rectum 36: 864-872, 1993.

8. Peeters KC, Marijnen CA, Nagtegaal ID, Kranenbarg EK, Putter H, Wiggers T, Rutten H, Pahlman L, Glimelius B, Leer JW, et al: The TME trial after a median follow-up of 6 years: Increased local control but no survival benefit in irradiated patients with resectable rectal carcinoma. Ann Surg 246: 693-701, 2007.

9. Swedish Rectal Cancer Trial, Cedermark B, Dahlberg M, Glimelius B, Påhlman L, Rutqvist LE and Wilking N: Improved survival with preoperative radiotherapy in resectable rectal cancer. N Engl J Med 336: 980-987, 1997. 
10. Bosset JF, Calais G, Mineur L, Maingon P, Radosevic-Jelic L, Daban A, Bardet E, Beny A, Briffaux A and Collette L: Enhanced tumorocidal effect of chemotherapy with preoperative radiotherapy for rectal cancer: Preliminary results-EORTC 22921. J Clin Oncol 23: 5620-5627, 2005.

11. Nakafusa Y, Hirohashi Y, Tanaka T, Kitajima Y, Sato S and Miyazaki K: Lateral lymph node dissection in treatment for advanced lower rectal cancer. Jpn J Gastroenterol Surg 34 $1512-1521,2001$.

12. Ishii Y, Hasegawa H, Endo T, Okabayashi K, Ochiai H, Moritani K, Watanabe M and Kitagawa Y: Medium-term results of neoadjuvant systemic chemotherapy using irinotecan, 5-fluorouracil, and leucovorin in patients with locally advanced rectal cancer. Eur J Surg Oncol 36: 1061-1065, 2010.

13. Hasegawa J, Nishimura J, Mizushima T, Miyake Y, Kim HM, Takemoto H, Tamagawa H, Noura S, Fujii M, Fujie Y, et al: Neoadjuvant capecitabine and oxaliplatin (XELOX) combined with bevacizumab for high-risk localized rectal cancer. Cancer Chemother Pharmacol 73: 1079-1087, 2014.

14. Schrag D, Weiser MR, Goodman KA, Gonen M, Hollywood E, Cercek A, Reidy-Lagunes DL, Gollub MJ, Shia J, Guillem JG, et al: Neoadjuvant chemotherapy without routine use of radiation therapy for patients with locally advanced rectal cancer: A pilot trial. J Clin Oncol 32: 513-518, 2014.

15. Kamiya T, Uehara K, Nakayama G, Ishigure K, Kobayashi S, Hiramatsu K, Nakayama H, Yamashita K, Sakamoto E, Tojima Y, et al: Early results of multicenter phase II trial of perioperative oxaliplatin and capecitabine without radiotherapy for high-risk rectal cancer: CORONA I study. Eur J Surg Oncol 42: $829-835,2016$
16. Hasegawa S, Goto S, Matsumoto T, Hida K, Kawada K, Matsusue R, Yamaguchi T, Nishitai R, Manaka D, Kato S, et al: A multicenter phase 2 study on the feasibility and efficacy of neoadjuvant chemotherapy without radiotherapy for locally advanced rectal cancer. Ann Surg Oncol 24: 3587-3595, 2017.

17. Rödel C, Grabenbauer GG, Papadopoulos T, Hohenberger W, Schmoll HJ and Sauer R: Phase I/II trial of capecitabine, oxaliplatin, and radiation for rectal cancer. J Clin Oncol 21: 3098-3104, 2003.

18. Tulchinsky H, Shmueli E, Figer A, Klausner JM and Rabau M: An interval $>7$ weeks between neoadjuvant therapy and surgery improves pathologic complete response and disease-free survival in patients with locally advanced rectal cancer. Ann Surg Oncol 15: 2661-2667, 2008

19. Roh MS, Colangelo LH, O'Connell MJ, Yothers G, Deutsch M, Allegra CJ, Kahlenberg MS, Baez-Diaz L, Ursiny CS, Petrelli NJ and Wolmark N: Preoperative multimodality therapy improves disease-free survival in patients with carcinoma of the rectum: NSABP R-03. J Clin Oncol 27: 5124-5130, 2009.

20. Sakuyama N, Kojima M, Kawano S, Akimoto T, Saito N, Ito M and Ochiai A: Histological differences between preoperative chemoradiotherapy and chemotherapy for rectal cancer: A clinicopathological study. Pathol Int 66: 273-280, 2016. 